\title{
Full Endoscopic Passing the Cavity of the Facet Approach for Treatment of Lumbar Disc Herniation
}

\section{TO THE EDITOR}

In 2000, Yeung (1) used the YESS system (yeung endoscopic spine system) to treat lumbar disc herniation, with an operating method using an intradiscal technique (inside-out technique); the indication for its use is contained lumbar disc herniation and far lateral herniation. To treat a prolapsed or sequestered lumbar disc, in 2006, Hoogland et al (2) used the TESSYS system (transforaminal endoscopic spine system); the operating method was an extradiscal (outside-in) technique. With the TESSYS system, it is possible to widen the neuroforamen (foraminoplasty) in stages using special reamers. After the anterior and lateral parts of the superior articular process is reamed, access can be gained to the prolapsed or sequestered disc. In the TESSYS method, the puncturing reams the lateral and anterior parts of the superior articular process that enlarge the intervertebral foramen (foraminoplasty).However, patients with a huge herniation, or facet hypertrophy, are still difficult to treat. In our clinical practice, we found a new approach for treatment: passing the cavity of the facet approach for lumbar disc herniation. Here we present a typical case.

Our patients was a 41-year-old man with left leg radicular symptoms of roughly 4 years' duration. Magnetic resonance imaging (MRI) revealed an L5/S1 prolapsed lumbar disc herniation (Fig. 1 A,B). No stability was found

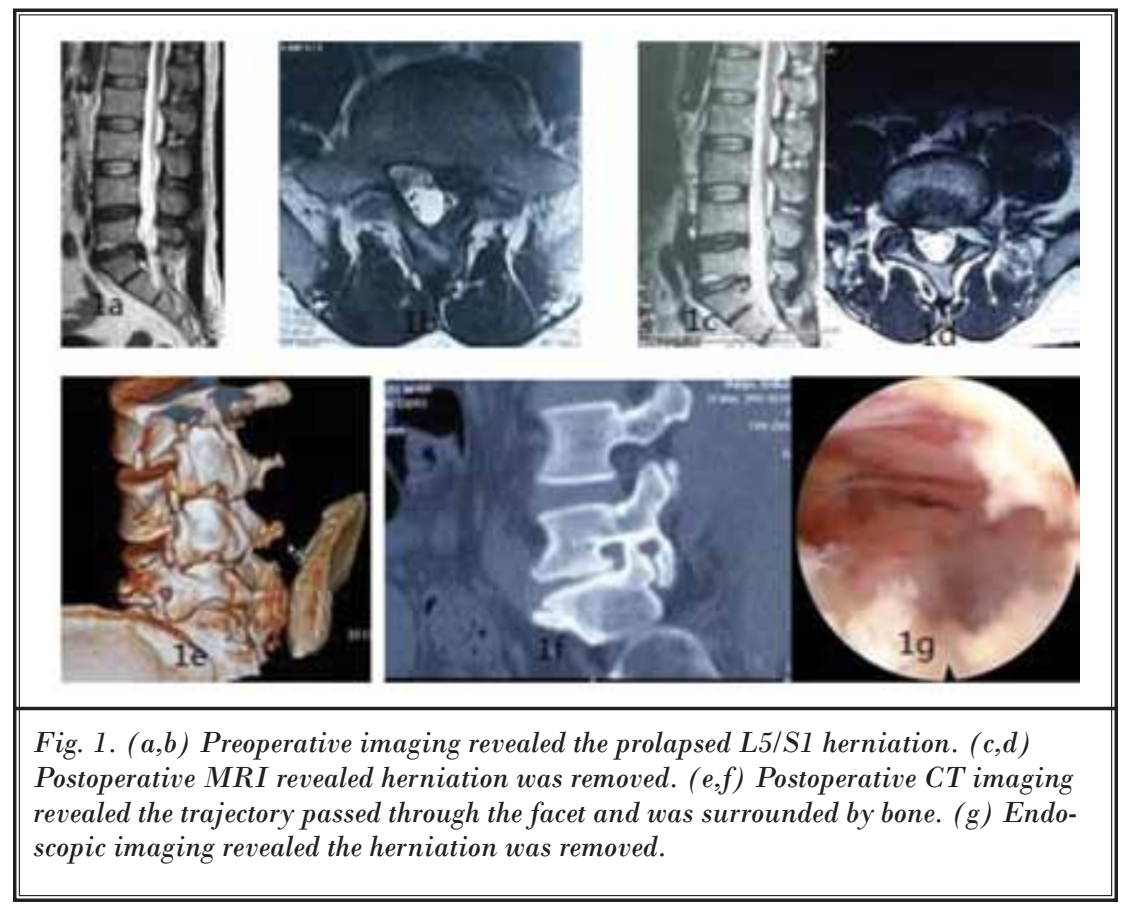

in extension and flexion lumbar radiographs. Postoperative MRI (Fig. 1 C,D) and computed tomography $(C T)$ (Fig. $1 \mathrm{E}, \mathrm{F}$ ) revealed a trajectory passing through the articular joint, and the prolapsed disc was removed. The endoscopic imaging revealed the prolapsed disc was completely removed and a good visualization of dural sac pulsation was achieved (Fig. $1 \mathrm{G}$ ).

The operation was generally carried out with the patient in the lateral decubitus position with the symptomatic side up. Lidocaine $1 \%$ was used as a local anesthetic. At the levels L3/4, $L 4 / 5$, and L5/S1, the posterior line of the inferior articular process in the lateral fluoroscopic view generally served as the safety line in order to avoid injury to the abdominal organs. The target point was the middle of the superior edge of the caudal vertebral body on the anteroposterior (AP) view, and the upper posterior margin of the caudal vertebral body on the lateral view (Fig. 2 A,B). Linking the tip of the superior articular process and the target point served as a puncturing line (Fig. 2 $A, B)$. The junction of the puncturing line and safety line was called the J point. The puncturing point was the J point or medial to the J point on the puncturing line. We always chose about $2 \mathrm{~cm}$ medial to the $J$ point as the puncturing point. We inserted the spinal needle into the articular cavity between the superior articular 

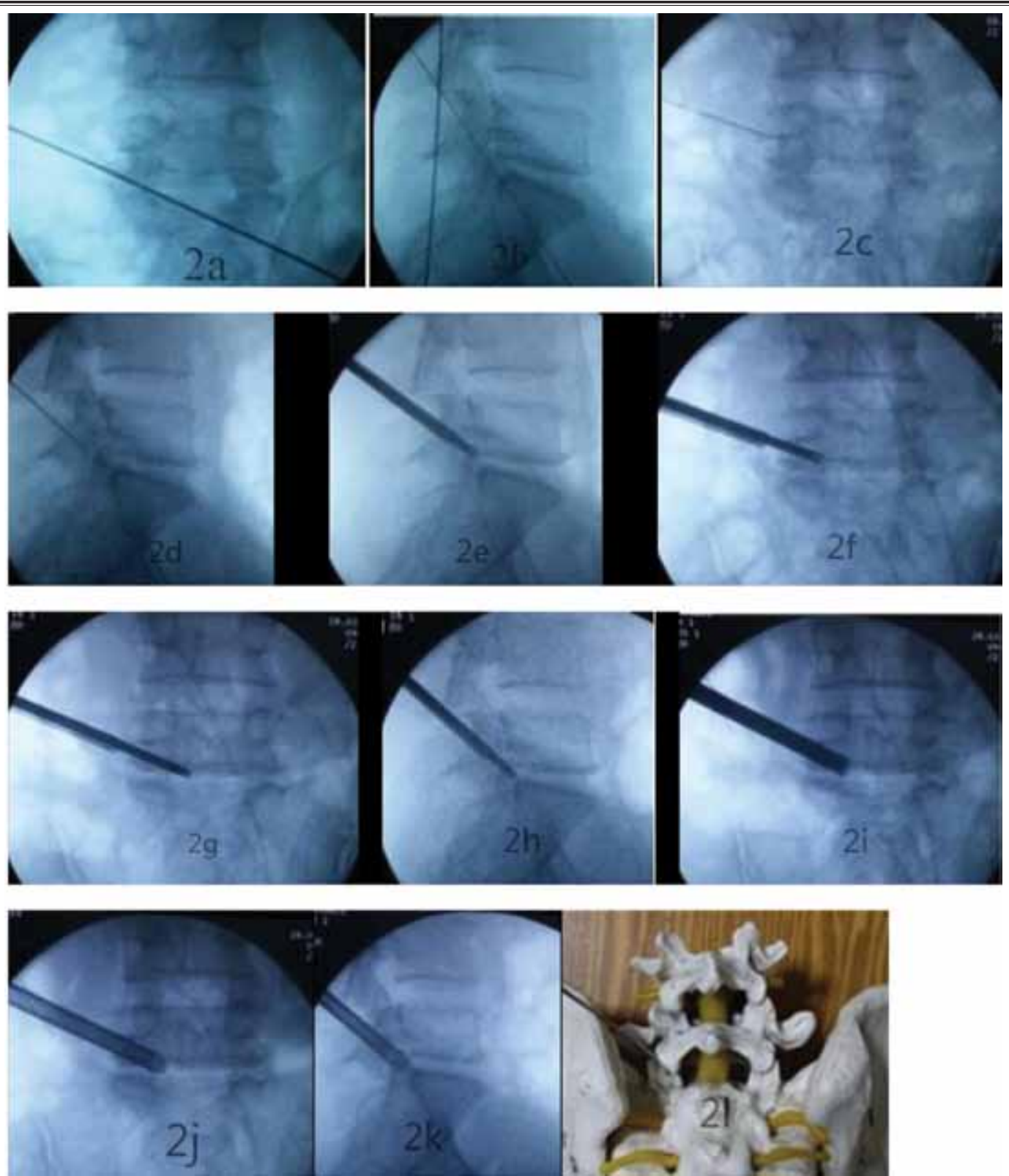

Fig. 2. (a,b) Preoperative imaging showing puncturing line and safety line. (c,d) Spinal needle was inserted into the articular cavity between the superior articular process and the inferior articular process on the AP plane and lateral view. (e,f) Through the last remaining guide tube, along the thinnest rod (green), part of the facet joint was removed with the thinnest reamer (green) (g-i) The second rod (yellow) was advanced to the target point on the AP and lateral planes, the second reamer (yellow) reamed the facet. $(j, k)$ The AP and lateral fluoroscopic views showed the position of the beveled cannula. (l) The spinal needle was in the cavity of the joint.

process and inferior articular process on both the AP and lateral views (Fig. 2 C,D). The wire was inserted. The thinnest rod (green) was advanced along the wire as far as the cavity of the facet joint. Subsequently, 3 further guide tubes of increasing diameter were advanced over the guide rod to widen the soft tissue channel. The first 2 guide tubes were removed. Through the last remaining guide tube, along the thinnest rod (green), part of 
the facet joint was removed with the thinnest reamer (green). The reaming procedure was visualized using fluoroscopy in 2 planes (Fig. 2 E,F). The reamer was not advanced beyond the medial pedicular line on the AP view. After reaming, the second rod (yellow) was advanced to the target point on AP and lateral views, and the second reamer (yellow) reamed the facet (Fig. $2 \mathrm{G}-\mathrm{I})$. With the same method, the largest rod was advanced, and the largest reamer (red) reamed the facet. Finally, the working cannula was inserted (Fig. 2 J,K). The prolapsed lumbar disc was removed through the endoscope and the nerve was decompressed.

In 1991, Kambin (3) described the "safe triangle": the hypotenuse is the exiting nerve root, the base is the superior border of the caudal vertebrae, and the height is the dura and the traversing nerve root, posteriorly by the superior articular process of the caudal vertebrae. In 2014, Kubaszewski et al (4) first presented a transfacet approach. They reported 3 older patients with L5/ S1 extruded disc herniations who underwent decompression by the foraminoplasty transfacet approach. In their technique, the guidewire was placed against the lower part of the intervertebral foramen and the approach trajectory was drawn to enter the center of the L5/S1 intervertebral disc on both the AP and lateral planes.

What we do differently from Kubaszewski is that we pass through the cavity of the facet obliquely in the superior to inferior direction (Fig. 2 I) and the trajectory is drawn to enter the middle of the superior edge of the caudal vertebral body on the AP view and the upper posterior margin of the caudal vertebral body on the lateral view. The advantage is that prolapsed or sequestered disc fragments can be easily removed from the spinal canal. In our technique, the trajectory is surrounded by the bone of the facet (Fig. $1 \mathrm{E}, \mathrm{F}$ ). If the anterior and lateral parts of the superior articular process are partially preserved, then it can better preserve the stability of the spine. But the biomechanical influence of the transfacet approach to lumbar stability needs further study.

This method can be used in patients with a huge herniation and/or facet hypertrophy. For these patients, if we ream the anterior and lateral parts of the superior articular process, a greater extent of resection is needed.

\author{
Guang-Quan Zhang \\ Henan Provincial People's Hospital \\ (Zhengzhou University People's Hospital) \\ Yan-Zheng Gao \\ Henan Provincial People's Hospital \\ (Zhengzhou University People's Hospital) \\ Shu-Lian Chen \\ Henan Provincial People's Hospital \\ (Zhengzhou University People's Hospital) \\ Shuai Ding \\ Henan Provincial People's Hospital \\ (Zhengzhou University People's Hospital) \\ E-mail 13837108751@163.com
}

\section{References}

1. Yeung AT. The evolution of percutaneous spinal endoscopy and discectomy: State of the art. Mt Sinai J Med 2000; 67:327332 .

2. Hoogland T, Schubert M, Miklitz B, Ramirez A. Transforaminal posterolateral endoscopic discectomy with or without the combination of a low-dose chymo- 4 . papain: A prospective randomized study in 280 consecutive cases. Spine (Phila Pa 1976)2006; 31:E890-E897.

3. Kambin P. Arthroscopic microdiskectomy. Mt Sinai J Med 1991; 58:159-164.
Kubaszewski L, Kaczmarczyk J, Nowakowski A, Sulewski A. Foraminoplastic transfacet epidural endoscopic approach for removal of intraforaminal disc herniation at the $\mathrm{L}_{5}-\mathrm{S}_{1}$ level. Wideochir Inne Tech Maloinwazyjne 2014; 9:96-100. 